\title{
THE INVESTIGATION OF OPEN CLUSTERS BY MEANS \\ OF THEORETICAL HR-DIAGRAMS *
}

(Abstract)

\author{
O. B. DLUZHNEVSKAYA and A. E. PISKUNOFF
}

Astronomical Council of the Academy of Science, U.S.S.R.

\begin{abstract}
An investigation of the initial mass function for 19 open clusters of different ages was carried out. The membership of stars in clusters was determined by means of UBV-photometry data, proper motions or radial velocities. The masses of cluster members were estimated using Paczyński's (1970) evolutionary sequences isochrones (Dluzhnevskaya et al., 1971) and B.C. and $T_{e}$ scales given by Johnson.

Assuming that the mass function may be expressed as $f(\mathfrak{M})=a \mathfrak{M}^{-\alpha}$ the values of ' $a$ ' and ' $\alpha$ ' were derived for each cluster. It is shown that ' $\alpha$ ' is an increasing function of the cluster age, while ' $a$ ' correlates with the cluster mass.

This may be interpreted as an evidence of certain evolutionary changes in the initial mass functions for stars with masses larger than $8 \mathfrak{M}_{\odot}$ in the solar vicinity.
\end{abstract}

\section{References}

Dluzhnevskaya, O. B., Musylev, V. V., and Rodionova, G. G.: 1971, Nauch. Inform. Moscow 21. Johnson, H. L.: 1966, Ann. Rev. Astron. Astrophys. 4, 93.

Paczyński, B.: 1970, Acta Astron. 20.

* This paper was presented by O. B. Dluzhnevskaya. 